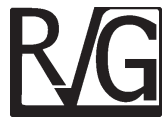

\title{
Gobierno corporativo y profesionalización empresarial en hipermercados y supermercados familiares en Venezuela
}

\author{
Hernández Fernández, Lissette* \\ Portillo Medina, Rafael** \\ Romero Borré, Jenny*** \\ Hernández Chacín, Ana****
}

\section{Resumen}

La empresa familiar es reconocida por su importante presencia en el mundo, caracterizada por una estructura en la que se conjugan dos sistemas con lógicas distintas: la familia y la empresa; razón por la que uno de sus retos es lograr el balance entre los intereses y objetivos de la empresa y la familia. El objetivo del presente artículo es describir el tipo de estructuras, planes y políticas de gobierno corporativo con el que operan los hipermercados y supermercados familiares en Venezuela. La investigación es no experimental de campo, con un tipo de estudio descriptivo, para lo que se utilizó un cuestionario. Entre los principales resultados: a) en los hipermercados y supermercados familiares las estructuras de gobierno formalizadas son las vinculadas al sistema de propiedad, y en contraposición se observa como las estructuras relacionadas con el sistema de familia son poco recono-

Recibido: 30-01-14 Aceptado: 10-12-14

* Doctora en Ciencias Humanas. Magíster en Gerencia de Empresas - Mención Financiera. Magíster en Economía-Mención Macroeconomía y Política Económica. Economista. Docente-Investigadora adscrita al Instituto de Investigaciones de la Facultad de Ciencias Económicas y Sociales de la Universidad del Zulia (LUZ) (Venezuela). Actualmente en estancia de investigación en la Universidad de la Costa (CUC) (Barranquilla, Colombia). Email: lissettehf@yahoo.es

** Magíster en Gerencia de Empresas-Mención Financiera. Especialista en Planificación. Economista. Docente-Investigador de la Facultad de Ciencias Económicas y Sociales de la Universidad del Zulia (LUZ) (Venezuela), y en la Universidad de la Costa (CUC) (Barranquilla, Colombia). Email: rportillomedina@gmail.com

*** Doctora en Ciencias Humanas. Magíster en Gerencia de Empresas - Mención Financiera. Economista. Docente-Investigadora adscrita al Instituto de Investigaciones de la Facultad de Ciencias Económicas y Sociales de la Universidad del Zulia (LUZ). Email: jennyfrb@yahoo.com

**** Economista. Auxiliar de investigación en el Instituto de Investigaciones de la Facultad de Ciencias Económicas y Sociales de la Universidad del Zulia (LUZ). Email: hernandezanaemilia@gmail.com 
cidas e implementadas; b) la planificación de la empresa figura como una categoría escasamente considerada y las políticas diseñadas son las relacionadas a las estructuras implantadas; c) Las estructuras de gobierno constituyen las plataformas para abordar y gestionar los aspectos relativos a los sistemas familia, empresa y propiedad, de forma constructiva y por separado, evitando solapamientos, o que siempre prime un sistema sobre el resto. Por ello, se concluye que se hace prioritario, potenciar y/o establecer mecanismos efectivos de gobierno en los tres ámbitos.

Palabras clave: Empresa familiar, gobierno corporativo, profesionalización empresarial, hipermercados, supermercados.

\section{Corporative Government and Entrepreneurial Professionalization in Family Hypermarkets and Supermarkets in Venezuela}

\section{Abstract}

The family business is recognized for its significant presence in the world, characterized by a structure in which two systems with different types of logic are joined: family and business. For this reason, one of its challenges is to achieve a balance between the interests and objectives of the business and the family. The objective of this article is to describe the type of structures, plans and policies of corporative government with which family hyper and supermarkets operate in Venezuela. This is non-experimental, field research with a descriptive type of study, for which a questionnaire was utilized. The main results include: a) in family hyper and supermarkets, the formalized structures of government are connected to the system of property, and in counter position, one can see how structures related to the family system are little recognized and implemented; b) planning the business figures as a category that is hardly considered, and the policies designed are related to the implanted structures; c) government structures constitute the platforms for approaching and managing the aspects relative to family, business and property systems, in a constructive manner and separately, avoiding overlapping or that one system is always favored over the rest. For these reasons, conclusions are that it is a priority to empower and/or establish effective government mechanisms in the three spheres.

Key words: Family business, corporative government, entrepreneurial professionalization, hypermarkets, supermarkets.

\section{Introducción}

La empresa familiar constituye uno de los pilares básicos de las economías de los países, por su rol como generadora de riqueza; dado su contribución al Producto Nacional Bruto (PNB); y, por su capacidad de empleadora. Se estima que entre el $65 \%$ y el $85 \%$ de las empresas del mundo son empresas familiares (Gersick et al, 1997); y su contribución al empleo varía según los países, generando como mínimo un $60 \%$ de los puestos de trabajo en las distintas economías.

En EEUU, según el ranking de la Revista Fortune, el $40 \%$ de las 500 empresas más grandes es de carácter familiar (Harvard Business School Bulletin, 1990: 78. En Ginebra, 2005); mientras que en España, el Instituto de Empresa Familiar señala que el $17 \%$ de las 1.000 mayores empresas son familiares (Ruzo et al, 2007). Asimismo, en los trabajos de 
Gobierno corporativo y profesionalización empresarial en hipermercados... Hernández, Lissette; Portillo, Rafael; Romero, Jenny y Hernández, Ana

Monreal y Sánchez (2012); Corona (2005); Gómez-Betancourt (2006); y Dodero (2008), se presenta una realidad similar en otros tantos países, con una presencia abrumadoramente mayoritaria de empresas familiares en las pequeñas y medianas empresas.

Una empresa familiar no es diferente a cualquier otra organización, salvo en un aspecto muy importante: la propiedad o el control de la misma está en manos de una familia o asociación de familias; y por lo general, son los miembros de ellas los que ocupan los puestos decisivos de la compañía. Es precisamente esta característica la que otorga un sello distintivo y conlleva a discriminar en empresas familiares y empresas no familiares. La dimensión familiar, otorgada a través de la participación en la propiedad y en la gestión, diferencia a estas empresas familiares de todas las demás y hace de este tipo de organización una institución emocional, ya que su gerencia y toma de decisiones es permeada por la afectividad derivada de trabajar con miembros de la familia, de modo que el fundador puede verse confundido cuando los valores y objetivos de la empresa y la familia; muchas veces contradictorios; se solapan. Las normas de la familia en cuanto a selección de personal, compensación económica, valoración de los individuos y formación de los mismos, no coinciden con las normas de la empresa en general, cuyo objetivo es la creación de valor y beneficios, y esto afecta inevitablemente a la estructura de este tipo de organizaciones.

El hecho de dirigir una empresa, cuya propiedad es de la familia, en la que participan miembros de ésta en su gestión y funcionamiento, genera los solapamientos entre los diferentes subsistemas que la integran (familia-empresa-propiedad), y la confusión en la toma de decisiones, generalmente son más emocionales que racionales, dado los diferentes roles que se desempeñan en la empresa.

Por esa razón se afirma que la familia es ante todo una institución emocional, por ello, los negocios familiares son una mezcla de sentimientos familiares, y necesidades empresariales (Amat, 2004; Corona, 2005 y Dodero, 2008). Los niveles de confianza y lealtad existentes dentro de la familia afectan el desarrollo del negocio, haciéndolos, con gran frecuencia, ineficientes en sus respuestas al mercado.

La realidad demuestra que en la empresa familiar privan, muchas veces, los compromisos familiares por sobre las exigencias del mercado y de la actividad propia de la empresa. El rigor, la reflexión en la toma de decisiones, la implantación de códigos de buen gobierno en el funcionamiento interno de las empresas familiares, sucumben con más frecuencia de la deseada al peso de los compromisos familiares.

Como respuesta a este comportamiento, múltiples autores (Cromie et al, 2001; León-Guerrero et al, 1998; Astrachan y Kolenko, 1994; Aragón et al, 2005; Gersick et al, 1997; Amat, 2004; Amat et al, 2008; Tadeo Basco, 2005; y Bosch Sans, 2006), visualizan la profesionalización de la empresa familiar, como una vía para mitigar o eliminar, en algunos casos, el conflicto emocional de trabajar con la familia, y responder oportunamente a las 
demandas del mercado (clientes y proveedores), alcanzando mayores niveles de competitividad.

La profesionalización empresarial es un proceso que propicia la creación de las estructuras de gestión y gobierno empresarial y familiar, así como la formalización de planes, políticas y prácticas que regulen las relaciones entre los individuos que integran los diferentes subsistemas (empresa, familia y propiedad) en las empresas familiares; generando las competencias para adaptarse a los cambios del mercado y ser competitivos (Hernández, 2009).

Por tanto, para que una empresa familiar alcance su profesionalización empresarial, y con ésta se logre mitigar los conflictos originados por el solapamiento de objetivos e intereses entre los sujetos situados en uno u otro subsistema, tendrá que trabajar en pro de la creación e institucionalización de las estructuras de gestión y de gobierno empresarial $y$ familiar.

El presente artículo hace énfasis en las estructuras de gobierno de la familia (asamblea y/o consejo de familia, protocolo familiar, plan de sucesión) y de la empresa (junta de accionistas o propietarios, consejo de administración y dirección). De allí que tenga como objetivo describir el tipo de estructuras, planes y políticas de gobierno corporativo con el que operan las cadenas de hipermercados y supermercados familiares venezolanos, como categoría o dimensión fundamental de la profesionalización empresarial.

Al respecto, algunos autores definen la profesionalización de la empresa a través de la profesionalización del gobier- no, argumentando la necesidad de mantener reglas claras para evitar los conflictos internos entre los diferentes sistemas que constituyen la organización (Astrachan y Kolenko, 1994; Aragón et al, 2005; Amat, 2004; Tadeo Basco, 2005).

La visión generalizada confirma que los sistemas de gobierno constituyen la línea fronteriza de las relaciones entre la familia y la empresa (Dodero, 2008), y son diseñados para salvaguardar los intereses a largo plazo de los accionistas, asegurando el crecimiento, la continuidad y el bienestar de los propietarios actuales y futuros (Lansberg, 2000). Los órganos de gobierno son formaciones y estructuras internas que imprimen valor a la empresa familiar constituyendo el núcleo a través del cual, formal o informalmente, se expresan las directrices generales estratégicas y de funcionamiento (Gallo y Amat, 2003).

Para el desarrollo de la investigación, cuyos resultados dieron origen al presente artículo, se siguió el enfoque epistemológico empírico-inductivo, con un diseño de investigación no experimental de campo, y un tipo de investigación descriptivo, empleando como población objeto de estudio, por razones históricas, económicas y sociales, las cadenas de hipermercados y supermercados familiares en Venezuela.

En relación a las razones históricas, económicas y sociales determinantes en la selección de la población, es pertinente señalar, que las empresas de distribución comercial de bienes de consumo masivo (retail), de las que forman parte las cadenas de hipermercados y supermercados, constituyen el sexto sector de la economía, reconocido como un sec- 
Gobierno corporativo y profesionalización empresarial en hipermercados... Hernández, Lissette; Portillo, Rafael; Romero, Jenny y Hernández, Ana

tor vital para crear nuevas posibilidades de trabajo, con importantes oportunidades en las categorías como el medio tiempo o el trabajo temporal (Bermúdez, 2007). Además de considerarse actores centrales de la industria de distribución de alimentos.

En ese sentido, cualquiera que sea la evolución o comportamiento de los hipermercados y supermercados tendrá su respectivo impacto sobre el resto de actores que integran la cadena. Esto viene dado por la interacción con la demanda y los hábitos de consumo alimentario (encadenamientos hacia delante, con los clientes); como por sus encadenamientos hacia atrás, con los productores agropecuarios, intermediarios locales y empresas comerciales, agroindustrias e industria de alimentos; y los encadenamientos hacia el lado, a través de los canales comerciales tradicionales en las cadenas agroalimentarias.

Es precisamente, este entramado de relaciones entre los hipermercados y supermercados con el sistema agroalimentario lo que resalta la importancia económica y social de este sector en el aparato productivo de las economías.

Asimismo, para el caso particular de las empresas familiares en Venezuela, es fundamental destacar que las cadenas de hipermercados y supermercados familiares constituyen uno de los secto- res cuya historia y tradición los une a una familia o asociación de familias emprendedoras, cuyas iniciativas datan, algunas, desde los años 40. Esta tendencia es análoga para aquellas empresas con las cuales mantiene los encadenamientos hacia atrás, productores agropecuarios, intermediarios locales, empresas comerciales, agroindustrias e industria de alimentos, los cuales mayoritariamente son empresas de propiedad familiar (Hiperventas, 2003a y 2003b). Por tanto, cualquiera que sea la política o acción que se inicie para el fortalecimiento de las cadenas de hipermercados y supermercados, emprendida por iniciativa de las empresas o como resultado de políticas públicas, derivará beneficios para el resto de actores con éstos vinculados o relacionados (hacia delante, hacia atrás y hacia el lado) a través del sistema agroalimentario.

Para la determinación de la población objeto de estudio se emplearon los siguientes criterios o intenciones: 1. Para cumplir con la característica de ser una empresa familiar se utilizó la definición aportada por Cabrera y García (1999) ${ }^{1}$; 2. Para la selección de las empresas de distribución comercial de bienes de consumo masivo, en el formato de hipermercados y supermercados, cuya actividad $u$ operaciones las realicen dentro del territorio venezolano, se acudió a la base de

La empresa familiar es aquella organización en donde la totalidad o la mayoría de las acciones está en manos de una familia o asociación de familias; los miembros de la familia participan de un modo activo en la gestión de la empresa asumiendo puestos de diversa responsabilidad; y en donde se ha producido, se están produciendo o se prevé que se va a producir en el futuro una transferencia de la empresa a un miembro de la siguiente generación de esa familia (Cabrera y García, 1999). 
datos de empresas afiliadas a la Asociación Nacional de Supermercados y Afines (ANSA), que es la organización que en Venezuela agrupa las empresas de este ramo; y 3 . Se incorporó como criterio el número de sucursales o agencias, ya que éste, además de significar la posibilidad de operar en diferentes zonas y/o estados, le otorga la característica de solidez y permanencia en el mercado. Por tanto, se definió como criterio más de dos sucursales.

Con base en estos criterios, la definición de la población se efectuó en dos fases: En la primera, del directorio de la Asociación Nacional de Supermercados y Afines (ANSA) se extrajo las empresas de distribución comercial bajo el formato de hipermercados y supermercados, los cuales totalizaron cincuenta y seis (56) hipermercados y supermercados ${ }^{2}$. De éstos, con más de dos sucursales o agencias, se obtuvieron veintidós (22). En una segunda fase se inició su discriminación según fuese familiar $(F)$ ○ no familiar (NF), de la cual sólo resultaron quince (15) hipermercados y supermercados de carácter familiar con más de dos (2) sucursales o agencias.

Posteriormente, para la recolección de los datos relativos a las estructuras de gobierno corporativo se empleó la técnica de la encuesta, mediante el uso de un cuestionario semi-estructurado, como instrumento de recolección de información, el cual fue validado mediante la realización de una "prueba de jueces", constituido por un grupo de expertos, especialistas en "metodología de la investigación" y "dinámica de empresas familiares" de la Universidad del Zulia y de la Universidad Castilla de la Mancha - España. El cuestionario consta de un total de 13 preguntas o reactivos distribuidos en tres bloques del siguiente modo: el primer bloque esta dirigido a obtener información sobre la persona encuestada; el segundo bloque contempla las características generales de la empresa familiar; $y$ el tercer bloque se focaliza en estudiar los órganos y estructuras de gobierno corporativo con el cual operan las cadenas de hipermercados y supermercados familiares, indagándose sobre dimensiones, tales como: tipos de estructuras de gobierno corporativo y tipos de políticas y planes de gobierno corporativo.

Dado el tamaño de la población, representado por un número finito, accesible para su estudio, se tomo la decisión de realizar un "censo" 3 , razón por la cual se consideró el total de la población para su estudio o análisis a través de la aplicación del instrumento. La organización y procesamiento de la información obtenida a través del cuestionario se realizó mediante el uso del paquete estadístico SPSS, apoyando el empleo de técnicas estadísticas univariadas (tablas de frecuencias), bivariadas (tablas de frecuencia múltiples y de contingencia); y multivariadas, específicamente el análisis de clúster o conglomerado.

3 "El censo comprende el conteo completo de los elementos de una población u objetos de estudio" (Malhotra, 1997: 359). 


\section{La empresa familiar y su dinámica: modelos explicativos}

Los primeros trabajos en los que se considera la empresa familiar como temática objeto de investigación científica datan de las décadas de los sesenta y setenta; pero es a inicios de los ochenta cuando se empiezan a plantear modelos explicativos de la dinámica de dichas empresas, a continuación se presentan los dos principales modelos que han sido la base de las construcciones posteriores:

Davis y Stern (1980 en Gimeno, 2000 y 2012) describen la empresa familiar como fruto de la intersección de dos sistemas sociales, la familia y la empresa. La familia sería un sistema basado en las emociones, y la empresa un sistema racional y objetivo; es el denominado "modelo de los dos círculos" (Gráfico 1).

El primer sistema se inclina por brindar protección a los miembros de la propia familia y ser incondicional hacia ellos. Su valor máximo es el amor. El segundo pretende retribuir en función de las contribuciones de cada miembro y se rige por valores relativos a la productividad. Los subsistemas interactivos familia $y$ empresa son centrales en el análisis de los negocios familiares. Es posible analizar el comportamiento de la empresa como orientado a la tarea, que pretende la generación de beneficios económicos. Por el otro lado, al examinar el comportamiento del sistema familiar dentro de la empresa, se observa la gran influencia de las relaciones emocionales que constituyen el proceso familiar.
Gráfico 1

El modelo de los dos círculos

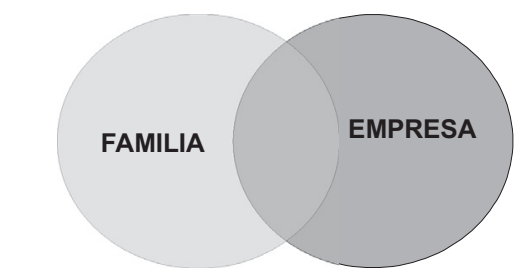

Fuente: Davis y Stern (1980 en Gimeno, 2000 y 2012).

Es posible visualizar un área de traslape entre círculos, que se considera un área de conflicto. El modelo ilustra que al realizarse una separación entre círculos (familia y empresa), la zona de conflicto disminuye. Esto significa que en la medida que se separen las cuestiones de familia de las de empresa, los conflictos se reducirán también.

En 1982, Tagiuri y Davis (En Gersick et al, 1997) introducen un segundo gran enfoque al definir el sistema de empresas familiares como subsistemas independientes pero superpuestos: el de la familia, el de la propiedad, el de la empresa. Este modelo es, probablemente, el que está actualmente más difundido con el nombre de "modelo de los tres círculos".

Según la lógica del modelo, cualquier miembro de este tipo de empresas puede ser ubicado en alguno de los siete sectores que forman los círculos al superponerse (Gráfico 2).

En el círculo "familia" se ubican todos y cada uno de los miembros del grupo familiar. Al círculo "empresa" pertenecen todos los individuos que laboran dentro de la misma y que perciben una remuneración por el trabajo que realizan para la empresa. El círculo "propiedad" está con- 


\section{Gráfico 2 \\ El modelo de los tres círculos}

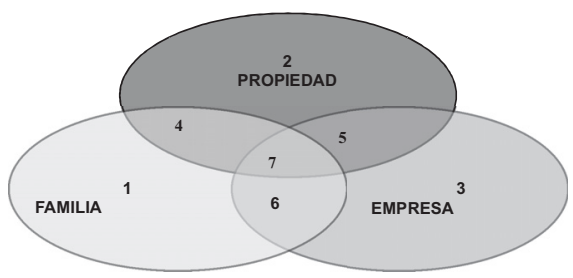

Fuente: Tagiuri y Davis (1982 en Gersick et al, 1997).

formado por los accionistas de la empresa. A partir de las intersecciones de estos tres círculos se origina un entramado socio-organizacional de importancia para la actuación de la empresa familiar (Gersick et al, 1997).

Así, todos los propietarios (socios o interesados en la empresa), y sólo ellos, se encontrarán en alguna parte del interior del círculo de la parte superior. De manera similar, todos los miembros de la familia se hallan en alguna parte del círculo izquierdo de la parte inferior y todos los empleados en el de la derecha. La persona que tenga sólo una conexión con la compañía estará en uno de los sectores externos: 1, 2 o 3. En base a lo anterior, el que tenga algún interés en la compañía y no sea miembro de la familia ni empleado irá en el sector 2 (dentro del círculo de la propiedad pero fuera de los dos restantes). Un miembro de la familia que no sea ni propietario ni empleado irá en el sector 1. Las personas que tengan más de un nexo con la organización se encontrarán en uno de los sectores sobrepuestos, que caen dentro de dos o tres de los círculos al mismo tiempo. Un propietario que además sea miembro de la familia pero que no sea empleado se hallará en el sector 4, que está dentro de los círculos de propiedad y de familia. Un propietario que trabaje en la empresa pero que no sea miembro de la familia aparecerá en el sector 5 . Finalmente, un propietario que además sea miembro de la familia y empleado estará en el sector central 7 , el cual se halla dentro de los tres círculos. Todo individuo que sea miembro del sistema de una empresa familiar ocupa un solo sitio en este modelo (Tagiuri y Davis, 1982. En Gersick et al, 1997).

El modelo de los tres círculos constituye una herramienta muy útil para entender la fuente de los conflictos interpersonales, dilemas de papeles, prioridades y límites de las empresas familiares. El hecho de especificar diversos papeles y subsistemas permite dividir las interacciones tan complejas dentro de la empresa familiar y además facilita ver lo que está ocurriendo y por qué (Gersick et al, 1997).

\section{Gobierno corporativo en empresas familiares: una aproximación teórica}

El gobierno corporativo o de la empresa se define como un sistema de procesos y estructuras para dirigir y controlar la empresa y responder por ello, así como para asegurar su viabilidad económica y su legitimidad (Neubauer y Lank, 1999 y 2003).

En ese sentido, se tiene que, dirigir significa participar en decisiones de naturaleza estratégica; controlar consiste en supervisar la labor de la dirección y vigilar el progreso hacia los objetivos (cómo se está implantando el plan estratégico, qué resultados se están obteniendo, entre otros); por responder entienden rendir 
Gobierno corporativo y profesionalización empresarial en hipermercados... Hernández, Lissette; Portillo, Rafael; Romero, Jenny y Hernández, Ana

cuentas a los que están legitimados para exigirlas en relación con la marcha de la empresa; asegurar la viabilidad económica radica en garantizar el desarrollo sostenible a largo plazo de la empresa, y, por último, la legitimidad de la empresa consiste en lograr su aceptación por la sociedad.

Cuando se trata de la empresa familiar, las relaciones entre los distintos grupos de interés involucrados con su actividad son más complejas, dado la presencia de un sistema adicional al de la empresa, como lo es el sistema familia, donde alguno (s) de sus miembro (s) pueden ser propietarios y/o empleados/directivos. Las relaciones que se establecen entre los accionistas o propietarios de la empresa, su consejo de administración, su alta dirección y cualquier otro grupo de interés que participe en la empresa son la fuente principal de sus potenciales conflictos.

Por esta razón, el gobierno corporativo tiene como principal objetivo limitar y resolver los problemas ocasionados por el conflicto de intereses que surgen entre los diferentes colectivos implicados directa o indirectamente en la actividad de la empresa. Con esta finalidad, en la empresa familiar se puede establecer un conjunto de órganos de gobierno que actúan como foros de discusión, de entendimiento mutuo y de resolución de conflictos.

Por tanto, las estructuras de gobierno corporativo constituyen una de las dimensiones o aspectos relevantes para que una organización logre profesionalizarse empresarialmente, con el fin de mantener reglas claras para evitar los conflictos internos entre los diferentes sistemas que constituyen la organización.
La profesionalización de la empresa pasa por alcanzar la profesionalización de la dirección y del gobierno. Los órganos de gobierno son formaciones y estructuras internas que imprimen valor a la empresa familiar constituyendo el núcleo a través del cual, formal o informalmente, se expresan las directrices generales estratégicas y de funcionamiento.

La profesionalización del gobierno hace referencia a la instauración de los órganos que regulan la acción entre el sistema familia y el sistema empresa. El gobierno de la familia está conformado por las asambleas y consejos de familia, constituyendo el foro donde se discuten la visión e intereses de la familia con respecto a la empresa; y el gobierno de la empresa contempla la asamblea de accionistas y el consejo de administración, garantes de los intereses a largo plazo de los accionistas.

Asimismo, la profesionalización de la dirección guarda relación con la creación de los órganos necesarios de dirección y gestión de la empresa (dirección general o comités directivos y gerencias), dotando a estos últimos de los sistemas y de las personas que desde su conocimiento y experiencia puedan desarrollar de forma profesional su trabajo. Estos órganos son los responsables de la orientación de la empresa, a través de la formulación de los planes estratégicos; así como de velar por su rentabilidad y desempeño.

Las estructuras, planes y políticas de gobierno corporativo que han sido empleados para explicar la dinámica de las empresas familiares (Gráfico 3) son los que a continuación se mencionan (Hernández, 2009 con base en Gersick et al, 1997): 


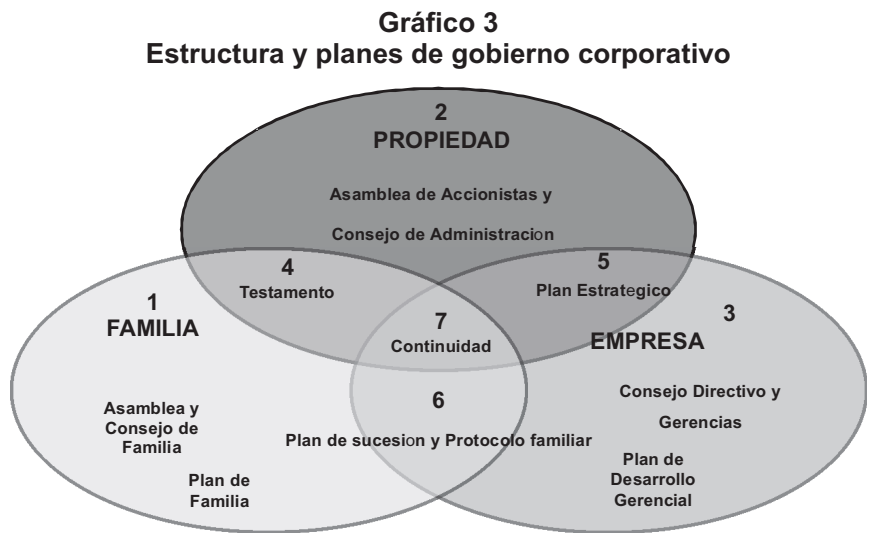

Fuente: Hernández (2009 con base en Gersick et al, 1997).

- En el sistema de familia: deben estar presentes la asamblea y/o consejo de familia; el plan de familia y las políticas de funcionamiento de la asamblea y/o consejo de familia.

- En el sistema de propiedad: debe disponerse de la asamblea de accionistas y el consejo de administración; y las políticas de funcionamiento del consejo de administración.

- En el sistema de la empresa: son relevantes el consejo directivo o los comités directivos y las gerencias en la organización; además el plan de desarrollo gerencial.

- Entre el sistema propiedad y el sistema familia: debe prepararse oportunamente el testamento.

- Entre el sistema propiedad y el sistema empresa: debe formularse el plan estratégico; y

- Entre el sistema familia y el sistema empresa: deben figurar el plan de sucesión y el protocolo familiar.

Los órganos de gobierno de la familia empresaria deben entenderse como instituciones de participación de los miembros de la misma en el devenir de la empresa, para garantizar su continuidad, reforzando los valores sobre los que se debe asentaren el futuro, así como para mantener la armonía, proporcionando cohesión y una visión compartida para alcanzar los objetivos de la organización.

Además, contribuyen en la toma de decisiones sobre las tareas que son responsabilidad de una familia empresaria, mantienen la fluidez de las relaciones personales, favoreciendo la comunicación, la participación, la toma de decisiones conjunta y la resolución de conflictos, y permiten educar a la familia y transmitir el legado y los valores de las generaciones anteriores.

Tanto la Asamblea como el Consejo de Familia se muestran como el cauce adecuado para solucionar aquellos problemas o conflictos que se vayan planteando en el seno familia.

La Asamblea de Familia es un órgano estrictamente para el funcionamiento del sistema familiar, que no debería entrar en cuestiones puramente empresa- 
Gobierno corporativo y profesionalización empresarial en hipermercados... Hernández, Lissette; Portillo, Rafael; Romero, Jenny y Hernández, Ana

riales. Tiene un carácter deliberante e informativo y puede ser de gran utilidad en una familia extensa. Está integrada por todos los miembros que hayan alcanzado una determinada edad. A través de este órgano se delibera sobre los intereses que se tiene en la empresa, sobre los objetivos que pretende conseguir, y el modo de participar en la gestión de la misma. Además de fomentar las relaciones entre los distintos miembros de la familia, así como detectar los posibles problemas que pudieran surgir en la misma y que pudieran tener repercusión en la empresa.

Por su parte, el Consejo de Familia es el órgano de carácter decisorio y debería estar integrado por representantes de todas las ramas y generaciones familiares, con independencia de que trabajen o no en la empresa. Sus reuniones deben ser periódicas y es deseable que exista un calendario de las mismas previamente fijado. La presidencia debería recaer en el miembro de la familia más respetado o en uno de los fundadores de la empresa, cualidades éstas que, en la práctica, suelen coincidir.

Sus principales funciones son: resolver aquellos problemas que se produzcan entre la familia y la empresa; dirigir y organizar la sucesión en la misma; defender los derechos de aquellos miembros de la familia que no trabajen en la firma; y diseñar, revisar y actualizar el protocolo familiar.

El protocolo familiar se constituye en un instrumento específico de las empresas familiares para regular todos aquellos aspectos que faciliten su continuidad, anticipándose a las eventuales discrepancias que puedan surgir en su seno. Es por tanto un acuerdo marco en- tre los miembros de la familia que regula: a) Las relaciones económicas y profesionales entre los miembros de ésta que ostentan la condición de socios y la propia empresa; y b) La gestión y organización de la empresa con el fin de asegurar la continuidad de la empresa.

El proceso de elaboración del protocolo familiar exige la colaboración de todos los miembros implicados y que, entre ellos, se realicen todos los debates necesarios sobre los temas a incluir en el mismo, ya que este proceso de discusión y posterior consenso hasta llegar al acuerdo final es clave para el eficaz funcionamiento de éste. Además, con el fin de adaptarlo a los cambios tanto familiares como empresariales que se vayan produciendo a lo largo de los años, es conveniente revisarlo periódicamente para mantener así su vigencia. Por lo tanto, un protocolo familiar ha de tener, de un lado, la vocación de permanencia en el tiempo y, de otro, el compromiso de examinarlo cada cierto tiempo.

Por tanto, el protocolo familiar debe ser un instrumento que sirva de norma para guiar las actuaciones familiares en la empresa y que, a su vez, sea capaz de contribuir a la solución de las eventuales incidencias que se produzcan en el seno de la familia, y que estén relacionadas con la organización.

Desde el subsistema de la propiedad, la Junta de Accionistas, es el órgano de gobierno vinculado a la propiedad de la sociedad mercantil, definido como el ente supremo o soberano de la sociedad, integrado por todos los socios de la misma. En la Junta se delibera y decide sobre los asuntos de la competencia de los accionistas o socios. A través de esta 
instancia los propietarios legales del negocio dejan explícitamente planteada la visión de la misma y la forma de gestionarlo.

Por su parte, el Consejo de Administración se configura como el órgano de gobierno asociado a la dirección de la empresa familiar, que tiene entre sus funciones la planificación y aseguramiento del proceso de sucesión en la dirección de la empresa; la intervención en la determinación de las estrategias de la empresa y el control de su compatibilidad con los intereses de la familia; la comunicación con los propietarios y familiares relacionados, directa e indirectamente con la empresa, y el establecimiento de la orientación estratégica y los objetivos a largo plazo; lo que contribuye en el proceso de profesionalización de los subsistemas que integran la empresa familiar. De esta manera, la existencia de estos dos órganos societarios permite diferenciar la propiedad y dirección de la empresa.

En el subsistema de la empresa, la Dirección o Comités de Dirección figuran ubicados en la cúspide del poder y responsabilidad, liderado por el director general. Son cargos de carácter ejecutivo, cuya misión es tomar decisiones, e implantar las directrices estratégicas establecidas por el consejo de administración. En este sentido, debe subrayarse la importancia de coordinar adecuadamente las labores de la alta dirección de la empresa y el consejo de administración cuando éste se dedica a desarrollar su función estratégica.

La alta dirección de la empresa familiar no debería diferir lo más mínimo de la de cualquier otra empresa. No obstante, en el caso de las empresas familiares surgen algunas peculiaridades, en el que destaca fundamentalmente la conveniencia de que el director general de la empresa pertenezca a la familia propietaria.

\section{Estructuras de gobierno corporativo en las cadenas de hipermercados y supermercados familiares en Venezuela}

\section{La descripción del tratamiento} otorgado a la estructuras, políticas y planes de gobierno corporativo en las cadenas de hipermercados y supermercados familiares en Venezuela, se realizó contemplando, en una primera instancia, el comportamiento común para las quince cadenas, determinado por las constantes; usando técnicas estadísticas univariadas (tablas de frecuencia) y bivariadas (tablas de frecuencias múltiple). Seguidamente, se trabajó con la conducta variable de las mismas, empleándose técnicas estadísticas multivariadas, específicamente el análisis de cluster o conglomerado, que permite agrupar las observaciones de forma que los datos sean homogéneos dentro de grupos (mínima varianza) y que estos grupos sean heterogéneos entre ellos (máxima varianza). En este caso, se utilizó como criterio para precisar la cantidad de conglomerados, el de la vinculación promedio (intergrupo), también conocido como de distancia media.

Mediante la aplicación del criterio de la vinculación promedio, se observa la formación de los subgrupos por etapas, que posteriormente, constituirán los dos conglomerados obtenidos de la población estudiada (Cuadro 1). 
Gobierno corporativo y profesionalización empresarial en hipermercados... Hernández, Lissette; Portillo, Rafael; Romero, Jenny y Hernández, Ana

\section{Cuadro 1}

Análisis de clúster

(Criterio de la vinculación promedio)

\begin{tabular}{cc}
\hline Grupo 1 & Grupo 2 \\
\hline S4 & H4 \\
S6 & S8 \\
H3 & S5 \\
H2 & S3 \\
S7 & S1 \\
H5 & H1 \\
H6 & \\
S9 & \\
S2 & \\
\hline
\end{tabular}

9 Cadenas de

6 Cadenas de

hipermercados $(\mathrm{H})$ y hipermercados $(\mathrm{H})$ y supermercados (S) supermercados (S)

Fuente: Hernández (2009).

\section{Como características generales} de las empresas bajo estudio figuran:

Las organizaciones incluidas en la población, en su totalidad, son grandes empresas, lo que se constató por el número de trabajadores, siguiendo el criterio aportado por el Instituto Nacional de Estadísticas (INE) y el contemplado por el Decreto con Rango, Valor y Fuerza de Ley para la Promoción y Desarrollo de la Pequeña y Mediana Industria y Unidades de Propiedad Social (2008), que consideran como gran empresa o industria aquella cuya nómina de empleados exceda los
100 trabajadores, tal y como se muestra en el Cuadro 2.

En ella se observa como en el $86,7 \%$ de las empresas (13 de éstas) la totalidad del capital es propiedad de una sola familia, y en el restante $13,3 \%$ la propiedad del capital está distribuida entre tres familias. Esta última situación convierte el proceso de toma de decisión más complejo, haciéndolo más lento, ya que en éste es necesario lograr un consenso entre los distintos propietarios.

También, se visualiza como estas empresas son dirigidas actualmente por la primera generación $(46,7 \%$ de las empresas, es decir, 7 empresas), y por la segunda generación un 53,3\% de las empresas (8).

Asimismo, se obtuvo como comportamientos comunes en torno a las estructuras, políticas y planes de gobierno corporativo en las cadenas de hipermercados y supermercados familiares en Venezuela, los siguientes (ver cuadros 3a, 3b y 3c). Entre los órganos y estructuras de gobierno con mayor presencia en las empresas estudiadas se tienen: La Junta de Accionistas, el Consejo de Administración y los Consejeros Externos, declarados por los empresarios en un $86,7 \%, 93,3 \%$ y $60 \%$, respectivamen-

\section{Cuadro 2}

Características generales de las cadenas de hipermercados y supermercados familiares en Venezuela

\section{Enunciado}

1. Número de empleados que posee la empresa

2. Composición del capital de la empresa

3. Generación familiar que dirige la empresa
Respuesta

Más de 100 empleados: 100,0\% (15)

Capital en una sola familia: $86,7 \%$ (13)

Capital distribuido en tres familias: $13,3 \%$ (2)

Primera generación: 46,7\% (7)

Segunda generación: $53,3 \%$ (8)

Fuente: Hernández (2009). 
te. De estos resultados se deriva como las estructuras de gobierno formalizadas son las vinculadas al sistema de propiedad, y en contraposición se observa como las estructuras relacionadas con el sistema de familia, son poco reconocidas e implementadas, como lo son las Asambleas y Consejos de Familia. El $86,7 \%$ de los empresarios declaró no poseerlas, comportamiento que puede constatarse a través del Cuadro $3 a$.

Asimismo, en relación a la planificación de la empresa se constató como el plan estratégico es formulado por el $86,7 \%$ de las organizaciones, el cual según Gersick et al (1997), permite plasmar los objetivos de los sistemas empresa y propiedad.

Por otra parte, el $86,7 \%$ de las empresas coincide en declarar definidas las políticas de funcionamiento del consejo de administración.
Cualquier otro tipo de instrumento de planificación no fue considerado por más del $80 \%$ de las organizaciones, entre estos destacan: el plan de familia (sistema familia); el testamento (sistema familia-propiedad); el plan de sucesión y el protocolo familiar (sistema familia-empresa), y el plan de desarrollo gerencial (sistema empresa).

A pesar de la relevancia que el plan de sucesión y el protocolo de familia, tienen como herramientas de planificación, no lo posee el $86,7 \%$ de las organizaciones, y sólo un $13,3 \%$ de éstas manifestó tenerlos elaborados; sin embargo, a manera de ilustración se presentan, a través de los Cuadros $3 b$ y $3 c$, los aspectos que contemplan estas herramientas, dado su importancia como medios que ayudan a definir las características y condiciones del sucesor, y a regular o normar la participación de los miembros de la familia en la empresa.

\section{Cuadro 3a \\ Cadenas de hipermercados y supermercados familiares en Venezuela: Órganos y estructuras de gobierno corporativo}

\section{Enunciado}

1. Existencia de las estructuras de - Asamblea de familia: No $86,7 \%$ (13) gobierno corporativo

- Junta de accionistas: Sí 86,7\% (13)

- Consejo de administración: Sí 93,3\% (14)

- Consejeros externos: Sí 60\% (9)

2. Existencia las siguientes políticas y planes

- Plan estratégico: Sí 86,7\% (13)
- Plan de desarrollo gerencial: No 86,7\% (13)

- Plan de familia: No 80\% (12)

- Plan de sucesión: No 86,7\% (13)

- Protocolo de familia: No $86,7 \%$ (13)

- Testamento: No 100\% (15)

- Políticas de funcionamiento del Consejo de Familia: No $86,7 \%(13)$

- Políticas de funcionamiento del Consejo de Administración: Sí 86,7\% (13) 
Gobierno corporativo y profesionalización empresarial en hipermercados... Hernández, Lissette; Portillo, Rafael; Romero, Jenny y Hernández, Ana

\section{Cuadro 3b}

Cadenas de hipermercados y supermercados familiares en Venezuela:

Órganos y estructuras de gobierno corporativo (Plan de sucesión)

\begin{tabular}{|c|c|}
\hline Enunciado & Respuestas \\
\hline $\begin{array}{l}\text { Plan de sucesión } \\
\text { en la empresa }\end{array}$ & $\begin{array}{l}\text { 1. Sólo lo poseen } 2 \text { empresas (13,3\%), y en su diseño } \\
\text { siguientes aspectos: } \\
\text { - Se especifica la forma de elección del sucesor } \\
\text { - Se establecen los requisitos que deben cumplir los pote } \\
\text { - Los planes sucesorios son consensuados por la familia } \\
\text { - Se establecen los mecanismos de entrada y entrenamie } \\
\text { - Generación preparada y motivada para promover el trá } \\
\text { - Se considera garantizado el gobierno futuro de la comp } \\
\text { dador no esté. } \\
\text { 2. Atributos exigidos al sucesor: } \\
\text { - Nivel de educación } \\
\text { - Experiencia en los negocios familiares } \\
\text { - Experiencia y habilidades financieras } \\
\text { - Emprendedor } \\
\text { - Integridad e inteligencia } \\
\text { - Compromiso con el negocio } \\
\text { - Habilidades para las relaciones personales } \\
\text { - Independencia y confianza en sí mismo } \\
\text { - Respetado por los empleados } \\
\text { - Afinidad con los objetivos y personalidad del líder } \\
\text { - Habilidad para la resolución de conflictos } \\
\text { - Respeto de los familiares empleados y no empleados } \\
\text { - Confianza de los familiares } \\
\text { - Relación de consanguinidad } \\
\text { - Edad del sucesor. }\end{array}$ \\
\hline
\end{tabular}

3. Tipo de sucesión del liderazgo que su empresa espera realizar: director o gerente miembro de la familia.

Fuente: Hernández (2009).

Los rasgos diferenciales de las estructuras, políticas y planes de gobierno corporativo, se derivaron a partir de la conformación de dos grupos o conglomerados, considerando para ello como variable los años de fundación de la empresa, característica fundamental para visualizar las conductas y actuaciones de las mismas.
Quedando los grupos constituidos de la siguiente manera:

El grupo 1 está integrado por nueve (9) cadenas de hipermercados y supermercados familiares ubicadas en el Distrito Capital, Zulia, Aragua, Nueva Esparta y Táchira, cuyos años de fundación se ubican en el período 1950-1999 y, por tanto, estas empresas se encuentran en la primera y segunda generación. 


\section{Cuadro 3c}

Cadenas de hipermercados y supermercados familiares en Venezuela:

Órganos y estructuras de gobierno corporativo (Protocolo familiar)

\begin{tabular}{|c|c|}
\hline Enunciado & Respuestas \\
\hline $\begin{array}{l}\text { Aspectos } \\
\text { contemplados en el } \\
\text { protocolo familiar }\end{array}$ & $\begin{array}{l}\text { - La misión y los valores de la familia } \\
\text { - Objetivos y valores de la empresa } \\
\text { - Objetivos del protocolo } \\
\text { - Órganos de gobierno en la familia y la empresa, y sus normas de funcio- } \\
\text { namiento } \\
\text { - Criterios para la incorporación de los accionistas en los órganos de go- } \\
\text { bierno } \\
\text { - El acceso a la condición de accionista } \\
\text { - Las políticas y normas laborales a seguir por los miembros familiares: } \\
\text { - incorporación, continuidad, permanencia y salida } \\
\text { - Las condiciones para la jubilación y la salida de la empresa de los gesto- } \\
\text { res } \\
\text { - Derechos de información contable de los miembros familiares } \\
\text { - Política de retribución de los miembros familiares en el consejo de familia } \\
\text { y en el consejo de administración } \\
\text { - La política de dividendos } \\
\text { - Los planes de sucesión y de contingencias } \\
\text { - La política de ayuda a familiares y a empleados no familiares } \\
\text { - La vigencia y las normas para la actualización del protocolo } \\
\text { - Las excepciones al protocolo familiar y la interpretación del mismo. }\end{array}$ \\
\hline
\end{tabular}

Fuente: Hernández (2009).

El grupo 2 está conformado por seis (6) cadenas de hipermercados y supermercados familiares localizadas en el estado Bolívar, Distrito Capital, y Zulia, cuyos años de fundación se encuentran en el período 1950-1979, razón por la cual en su mayoría $(83,3 \%)$ se ubica en la segunda generación, a excepción de un caso $(16,7 \%)$, que no se conoció el año de fundación.

La distribución de las empresas según los años de fundación y la generación familiar que la dirige, se puede observar a través de la Tabla 1.

En los tipos de estructuras de gobierno corporativo, se observan rasgos diferenciales, como lo es el caso de la presencia o existencia de los consejos de familia. Esta es una estructura típica del sistema de familia, que la posee el $22,2 \%$ y el $50 \%$ de las empresas del conglomerado 1 y 2 , respectivamente (Tabla 2).

Asimismo, se observa como las organizaciones que la han incorporado como estructura de gobierno de la familia, se caracterizan por ser las que tienen mayor antigüedad en ambos grupos, de allí que al haber transitado a la siguiente generación, o al estar en proceso para ésta, entiendan la importancia que este órgano reviste como mediador en las relaciones 
Gobierno corporativo y profesionalización empresarial en hipermercados... Hernández, Lissette; Portillo, Rafael; Romero, Jenny y Hernández, Ana

Tabla 1

Años de fundación. Generación familiar que dirige la empresa

\begin{tabular}{|c|c|c|c|c|c|}
\hline & \multicolumn{2}{|c|}{$\begin{array}{c}\text { Indique la generación } \\
\text { familiar que dirige la } \\
\text { empresa, teniendo en } \\
\text { cuenta la persona que } \\
\text { ostenta el cargo de más } \\
\text { alta jerarquia }\end{array}$} & \multirow[b]{2}{*}{ Total } \\
\hline & & & $\begin{array}{c}\text { 1era } \\
\text { generación } \\
\text { (Propietario- } \\
\text { Controlador) }\end{array}$ & $\begin{array}{c}2 \text { da } \\
\text { generación } \\
\text { (Sociedad de } \\
\text { Hermanos) }\end{array}$ & \\
\hline \multirow{12}{*}{$\begin{array}{l}\text { Años de } \\
\text { fundación }\end{array}$} & \multirow{2}{*}{ Entre 1950-1959 } & Recuento & & 4 & 4 \\
\hline & & $\%$ del total & & $26,7 \%$ & $26,7 \%$ \\
\hline & \multirow{2}{*}{ Entre 1960-1969 } & Recuento & & 3 & 3 \\
\hline & & $\%$ del total & & $20,0 \%$ & $20,0 \%$ \\
\hline & \multirow{2}{*}{ Entre $1970-1979$} & Recuento & 3 & 1 & 4 \\
\hline & & $\%$ del total & $20,0 \%$ & $6,7 \%$ & $26,7 \%$ \\
\hline & \multirow{2}{*}{ Entre 1980-1989 } & Recuento & 2 & & 2 \\
\hline & & $\%$ del total & $13,3 \%$ & & $13,3 \%$ \\
\hline & \multirow{2}{*}{ Entre 1990-1999 } & Recuento & 1 & & 1 \\
\hline & & $\%$ del total & $6,7 \%$ & & $6,7 \%$ \\
\hline & \multirow{2}{*}{$\mathrm{Ns} / \mathrm{Nc}$} & Recuento & 1 & & 1 \\
\hline & & $\%$ del total & $6,7 \%$ & & $6,7 \%$ \\
\hline \multirow{2}{*}{\multicolumn{2}{|c|}{ Total }} & Recuento & 7 & 8 & 15 \\
\hline & & $\%$ del total & $46,7 \%$ & $53,3 \%$ & $100,0 \%$ \\
\hline
\end{tabular}

Fuente: Hernández (2009).

Tabla 2

Presencia del consejo de familia en la empresa según su año de fundación

\begin{tabular}{|c|c|c|c|c|c|c|c|c|c|c|c|c|}
\hline & & \multicolumn{5}{|c|}{ Años de fundación } & \multirow{2}{*}{$\begin{array}{c}\text { Total } \\
\text { Grupo } \\
1\end{array}$} & \multicolumn{4}{|c|}{ Años de fundación } & \multirow{2}{*}{$\begin{array}{c}\text { Total } \\
\text { Grupo } \\
2\end{array}$} \\
\hline & & $\begin{array}{l}\text { Entre } \\
1950- \\
1959 \\
\end{array}$ & $\begin{array}{c}\text { Entre } \\
1960- \\
1969 \\
\end{array}$ & $\begin{array}{c}\text { Entre } \\
1970- \\
1979 \\
\end{array}$ & $\begin{array}{c}\text { Entre } \\
1980- \\
1989 \\
\end{array}$ & $\begin{array}{c}\text { Entre } \\
1990- \\
1999 \\
\end{array}$ & & $\begin{array}{c}\text { Entre } \\
1950- \\
1959 \\
\end{array}$ & $\begin{array}{l}\text { Entre } \\
1960- \\
1969 \\
\end{array}$ & $\begin{array}{c}\text { Entre } \\
1970- \\
1979 \\
\end{array}$ & $\mathrm{Ns} / \mathrm{Nc}$ & \\
\hline $\begin{array}{l}\text { Consejo } \\
\text { de }\end{array}$ & Sí & & 1 & & & 1 & $\begin{array}{c}2 \\
(22,2 \%)\end{array}$ & 3 & & & & $\begin{array}{c}3 \\
(50 \%)\end{array}$ \\
\hline Familia & No & 1 & 1 & 3 & 2 & & $\begin{array}{c}7 \\
(77,8 \%)\end{array}$ & & 1 & 1 & 1 & $\begin{array}{c}3 \\
(50 \%)\end{array}$ \\
\hline Total & & 1 & 2 & 3 & 2 & 1 & $\begin{array}{c}9 \\
(100 \%)\end{array}$ & 3 & 1 & 1 & 1 & $\begin{array}{c}6 \\
(100 \%)\end{array}$ \\
\hline
\end{tabular}

Fuente: Hernández (2009).

entre los miembros familiares. Sin embargo, también se constata como en el grupo 1 despunta una empresa, que sin haber llegado a los 20 años de antigüedad, tuvo la visión de haber diseñado el consejo de familia.
Del Cuadro 3a, se deriva como otras de las estructuras de gobierno empleadas son las relacionadas con el área de la propiedad, entre las que figuran: la asamblea de accionistas y el consejo de administración o de directores. 


\section{Conclusiones}

La fundamentación teórica presentada y los resultados observados en la gestión de las cadenas de hipermercados y supermercados familiares en Venezuela son demostrativos que la definición de las estructuras de gobierno empresarial y familiar son inaplazables.

Las estructuras de gobierno (empresarial-familiar) proveen las plataformas para que los retos y preocupaciones específicos de la familia, la empresa y de los propios accionistas puedan ser tratados y gestionados de forma constructiva y por separado. Por ello destaca como prioridad potenciar y/o establecer mecanismos efectivos de gobierno en los tres ámbitos.

El desarrollo del buen gobierno, que pasa obligatoriamente por la definición y puesta en marcha de las estructuras correspondientes a los subsistemas (empresa - familia - propiedad), permite garantizar un funcionamiento adecuado del negocio familiar al posibilitar una definición clara del papel, los derechos y responsabilidades de los empleados, propietarios y miembros de la familia; alcanzar una clara separación de los ámbitos de tratamiento de las cuestiones y problemas específicos de cada grupo (asuntos relativos a la propiedad, a la familia y a la empresa) reduciendo el impacto de tensiones o problemas de un grupo en el resto de los grupos; y lograr la incorporación de la familia de una forma apropiada en el proceso de discusión y de decisión relativo a la empresa.

La presencia formal de estas estructuras contribuye a reducir la probabili- dad de tensiones y conflictos. Sin embargo, no basta con establecer órganos de gobierno formales para gestionar adecuadamente los posibles conflictos, es preciso lograr que las estructuras cuenten con los procesos necesarios y planes formales (v.g. protocolo familiar, plan de sucesión, plan de retiro, plan estratégico, plan de desarrollo gerencial) para su buen funcionamiento; $y$ que además sus miembros cuenten con la actitud y las capacidades necesarias para la resolución de conflictos.

Trabajando en las estructuras de gobierno y sus respectivos planes y políticas se contribuirá a la profesionalización empresarial, proveyendo a la organización de las capacidades necesarias, definidas como la habilidad para crear estructuras y procesos internos que influyan en sus miembros, de modo que creen competencias específicas para adaptarse a los cambios del mercado, logrando ser competitivos en relación al resto de empresas.

Las cadenas de hipermercados y supermercados familiares en Venezuela deben trabajar por formalizar sus estructuras de gobierno corporativo, que conjuntamente con las políticas de recursos humanos, los diseños organizativos y las tecnologías de información y comunicación (TICs), conforman las dimensiones o ámbitos a través de los cuales alcanzarán la profesionalización empresarial.

\section{Referencias bibliográficas}

Amat, Joan; Roure, Juan y Martínez, Jon (2008). Transformarse o desaparecer. Estrategias de la empresa familiar para competir en el siglo XXI. 
Gobierno corporativo y profesionalización empresarial en hipermercados... Hernández, Lissette; Portillo, Rafael; Romero, Jenny y Hernández, Ana

Editorial Deusto. Primera edición. Bilbao. España. Pp. 306.

Amat, Joan (2004). La continuidad de la empresa familiar. Ediciones Gestión 2000, S.A. Barcelona. España. Pp. 175

Aragón, Cristina; Iturrioz Cristina, Aranguren, Mari José y Olarte, Francisco (2005). La empresa familiar en Gipuzkoa. Cámara de Gipuzkoa. San Sebastián. España.

Asociación Nacional de Supermercados y Autoservicios (ANSA) (2008). Directorio de empresas inscritas. Julio. Caracas. Venezuela.

Astrachan, Joe y Kolenko, Thomas (1994). Un aspecto ignorado que explica el éxito de la empresa familiar: Las prácticas de recursos humanos. Family Business Review. Vol. 7. № 3. Family Firm Institute (FFI). pp. 251-262.

Bermúdez, Ulises (2007). Retail venezolano: presente y futuro. Revista Punto de Venta. ANSA. Año 7. No. 39. Junio-Agosto. http://www.ansa.org.ve/ (Recuperado el 30 de abril de 2008).

Bosch Sans, Vicenc (2006). Alta dirección y empresa familiar. Lupa Empresarial On Line. Asociación Catalana de Empresa Familiar. CEIPA. http://www. ceipa.edu.co/ceipa/subsitios/lupa_ empresarial/ediciones_online/alta _direccion.pdf (Recuperado el 10 de abril de 2008).

Cabrera, María Katiuska y García, Juan Manuel (1999). La empresa familiar: dimensiones conceptuales y perspectivas teóricas. Revista europea de dirección y economía de la empresa. Vol. 8. № 1. Academia Europea de Dirección y Economía de la Empresa (AEDEM) España. pp. 7-30.

Corona, Juan (2005). Manual de la empresa familiar. Ediciones Deusto. España. Pp. 513.
Cromie, Sam; Dunn, Beuce; Sproull, Allan; y Chalmers, David (2001). Small firms with a family focus in the Scottish Highlands and Islands. The Irish Journal of Management. Vol. 22. $\mathrm{N}^{\circ}$ 2. Irish Academy of Management. Irlanda. pp. 45- 66.

Dodero, Santiago (2008). El secreto de las empresas familiares exitosas. Editorial El Ateneo. Segunda edición. Buenos Aires. Argentina.

Gallo, Miguel Ángel y Amat, Joan (2003). Los secretos de las empresas familiares centenarias. Editorial Deusto. Barcelona. España. Pp. 228.

Gersick, Kelin; Davis, John; McCollom, Marion y Lansberg, Ivan (1997). Empresas familiares: Generación a generación. Editorial Mc GraW Hill. México, D.F.

Gimeno, Alberto (2012). Modelos de empresa familiar y la incorporación de sus futuras generaciones. Family Business Summit 2012. Junio. América Empresarial LTDA. Bogotá.

Gimeno, Alberto (2000). Reflexión sobre la Bibliografía de la Empresa Familiar. I Congreso Nacional de Investigación sobre la Empresa Familiar. Valencia, España.

Ginebra, Joan (2005). Las empresas familiares. Su dirección y su continuidad. Sexta reimpresión. Panorama Editorial S.A. México. México. Pp 247.

Gómez-Betancourt, Gonzalo (2006). ¿Son iguales todas las empresas familiares?. Caminos por recorrer. Grupo Editorial Norma. Primera edición. Bogotá. Colombia. Pp. 317.

Hernández, Lissette (2009). Construcción teórica de la profesionalización empresarial en organizaciones familiares. Cadenas de hipermercados y supermercados familiares en Venezuela. Tesis Doctoral. Doctorado en Ciencias Humanas. Facultad de Humanidades y 
Educación. Universidad del Zulia. Maracaibo. Venezuela.

Hiperventas (2003a). El Patio, tres historias, tres generaciones. Revista Hiperventas. http://www.hiperventas.com/ detalle. asp? Seccion=Historia\&id $=10$ 7212 (Recuperado el 15 de agosto de 2008).

Hiperventas (2003b). Plaza's, Apostando por la tecnología. Revista Hiperventas. http://www.hiperventas.com/detalle. asp?Seccion=Historia\&id=108 (Recuperado el 15 de agosto de 2008).

Lansberg, Ivan (2000). Governance as a competitive advantage. GEEF Congress: The Paris Conference of the European Owner - Managed and Family Business Group. Paris. June 7-9.

León-Guerrero, Anna; McCann, Joseph y Haley Jr. Jonathan (1998). A study of practice utilization in family businesses. Family Business Review. Vol. 11. N² 2. pp. 107-120.

Malhotra, Naresh (1997). Investigación de Mercados. Un enfoque práctico. (Trad. V. de Parres). México. Prentice-Hall (Original en inglés, 1996).

Monreal, Juan y Sánchez, Gregorio (Coord.). (2012). El éxito de la empresa fami- liar. La relación entre negocio y familia. Editorial Aranzadi, S.A. Primera edición. España. pp. 282.

Neubauer, Fred. y Lank Alden (2003). La empresa familiar. Cómo dirigirla para que perdure. Ediciones Deusto S.A. Tercera edición. Bilbao. España. pp. 315.

República Bolivariana de Venezuela (2008). Decreto con Rango, Valor y Fuerza de Ley para la Promoción y Desarrollo de la Pequeña y Mediana Industria y Unidades de Propiedad Social. Gaceta Oficial N $N^{\circ} 5.890$ Extraordinario. $31 \mathrm{de}$ Julio. Caracas. Venezuela.

Ruzo, Emilio; Lozada Fernando y Gómez, María (2007). El diseño organizativo de la empresa familiar gallega. "El comportamiento de la empresa ante entornos dinámicos". XIX Congreso anual y XV Congreso Hispano Francés de Asociación Europea de Dirección y Economía de Empresa (AEDEM). Vol. 1 (Ponencias). pp. 61.

Tadeo Basco, Rodrigo (2005). Comportamiento en la dirección y gobierno de la empresa familiar: Análisis empírico de la profesionalización como garantía de continuidad. Tesis Doctoral. Universidad Complutense de Madrid. España. 